\title{
Discrete Time Analysis of Cognitive Radio Networks with Saturated Source of Secondary Users
}

\author{
Attahiru S. Alfa ${ }^{1, \star}$, Vicent $\mathrm{Pla}^{2}$, \\ Jorge Martinez-Bauset ${ }^{2}$, and Vicente Casares-Giner ${ }^{2, \star \star}$ \\ 1 University of Manitoba, Canada \\ 2 Universitat Politècnica de València, Spain
}

\begin{abstract}
The strategy used for sensing in a cognitive radio network affects the white space that secondary users (SUs) perceive and hence their throughput. For example, let the average time interval between consecutive sensing be fixed as $\tau$. There are several possible ways to achieve this mean value. The SU may sense the channel at equal intervals of length $\tau$ or sense it at randomly spaced intervals with mean value $\tau$ and guided by, for example, geometric distribution, uniform distribution, etc. In the end the strategy selected does affect the available white space and throughput as well as the resources spent on sensing. In this paper we present a discrete time Markov chain model for cognitive radio network and use it to obtain the efficiency of sensing strategies. The system studied is one in which we have a saturated source of secondary users. These assumptions do not in any ways affect our results.
\end{abstract}

\section{Introduction}

Cognitive radio (CR) has been proposed as one approach to deal with the limited unlicensed spectrum availability. Most of the licensed spectrum are not fully utilized most of the time. Capturing the unused time slots, which are known as spectrum holes, on these available spectrum and using them efficiently is what $\mathrm{CR}$ is about. The FCC has approved the use of these licensed spectrum by unlicensed users when there is a spectrum hole, subject to a limited interference to the licensed users [2]. This spectrum hole we refer to as white space. The key to success for CR consists of effective and efficient sensing of the channels by the secondary users ( $\mathrm{SU})$, the unlicensed users, to detect white spaces when they occur. A decision making process then follows the sensing, based on the outcome of the sensing exercise.

\footnotetext{
* The research of this author was supported in part by the Spanish government through grant SAB2009-0132.

** The research of these authors was supported by the Spanish government through the projects TIN2008-06739-C04-02 and TIN2010-21378-C02-02, and by Universitat Politècnica de València through PAID-06-09.
} 
Each time an SU senses the channels, it expends some resources. A lot of resources are wasted if too many sensing episodes result in unsuccessful capture of a white space. On the other hand, infrequent sensing could lead to the inability to detect available white space to transmit messages, resulting in long delays. In the end a very good balance regarding the sensing strategy is critical in order to detect white space early enough in the sensing period with minimum sensing frequency. Despite advance research in the area of CR, little is known about theoretically founded method for determining the optimal sensing strategy. In this paper we develop a sensing strategy based on a discrete time Markov chain.

If we observe a PU channel, it is either busy or idle. The idle periods are the holes referred to as white space. The busy and idle periods form an alternating sequence of events which may be correlated. An SU sensing this channel at some time points either finds the channel busy or idle. When a sensing occurs during the idle time of a PU then the SU is able to effectively utilize the remaining idle time, which we call the effective white space. The ideal sensing strategy is one that results in the SU capturing most of the white space when it finds the channel to be idle, i.e. the one that can minimize the difference between the lengths of the white space and the effective white space, while keeping resource utilization to a minimum. As pointed out by Yücek and Arslan [9, selection of sensing parameters result in trade off between sensing frequency, reliability and the actual characteristics of the PU channel usage. So the aim in CR networks is to plan the sensing in order to utilize high proportion of the white space.

The sensing strategy affects the length of the effective white space and hence determines the efficiency of the strategy. The often made assumption is that sensing should be done periodically and the issue is determining the interval between the sensing times [4. However, there are no documented analytically based studies supporting the idea of using constant interval between sensing times as the optimal strategy. In addition, most models that have been developed for studying cognitive radio networks usually assume that the durations of both the busy and idle times are governed by the exponential distributions, see for example Lee and Akyilidz [6]. Geirhofer et al [3] were among the earlier researchers to show from measurements that the idle time duration was more of a lognormal distribution. This was further confirmed by Wellens et al [8] who showed that the idle time duration has lognormal distribution for long durations and geometric distribution for the short ones. Most of the previous works also ignored the possible correlation between the busy and idle times, even though logically one would expect some correlation to exist between the two intervals. Hoang et al [5] did consider such correlation by introducing a two-state Markov chain to capture this aspect. Our aim in this paper is to determine how to arrive at optimal sensing strategy under any cognitive radio network conditions, using representative mathematical models. We do this by representing the PU busy and idle periods as alternating Markov phase renewal process [1] and develop a model to determine effective white space under different sensing strategies. This Markov phase renewal process allows the busy and idle times durations to assume a wide variety of distributions and also captures a much broader correlation aspects of the two intervals. By sensing strategies we imply using different 
statistical distributions of intervals between sensing time points for a fixed mean sensing interval. From this we obtain the sensing efficiency which is practically the ratio of the lengths of the effective white space to that of the white space. The model presented in this paper is used as a base work for studying a cognitive radio network in detail in our future work.

The rest of the paper is organized as follows. Section 2 introduces the model of the channel as seen by the SU, i.e., the pattern of channel busy periods and white spaces. The behavior of the CR system is described in Section 3 . Then a Markov chain model of the system is developed and some performance measures are derived. In Section 4 we present a numerical study that evaluates the efficiency of different sensing strategies and the adjustment of their parameters. Finally, the paper is concluded in Section 5 .

\section{Characteristics of the Channel}

We observe our system in discrete time points $0,1,2, \cdots$. Consider a single communication channel used by a primary user, PU. Here the PU refers to the whole set of PUs which might use the channel under consideration. This channel is either in use (busy) or not in use (idle). Consider a discrete time Markov chain (DTMC) with state space $\left\{1,2, \cdots, n_{b}, n_{b}+1, \cdots, n_{b}+n_{i}\right\}$, wit the transition matrix representing this DTMC given as D. Let the PU busy (b) and idle (i) conditions be represented by the sets of states $\left\{1,2, \cdots, n_{b}\right\}$ and $\left\{n_{b}+1, \cdots, n_{b}+n_{i}\right\}$, respectively. Further define the substochastic matrices $D_{b}$ and $D_{i}$, to represent the transitions within the busy and idle periods, respectively, and $d_{b i}$ to represent transitions from busy to idle and $d_{i b}$ from idle to busy. The $D_{b}$ and $D_{i}$ are substochastic matrices of orders $n_{b}$ and $n_{i}$, respectively. By these definitions we have $D_{b} \mathbf{1}+d_{b i} \mathbf{1}=\mathbf{1}$ and $D_{i} \mathbf{1}+d_{i b} \mathbf{1}=\mathbf{1}$, where $\mathbf{1}$ is a column vector of ones. Based on this, the channel is either busy or idle according to the following transition matrix

$$
D=\left[\begin{array}{cc}
D_{b} & d_{b i} \\
d_{i b} & D_{i}
\end{array}\right] .
$$

Let $\boldsymbol{\pi}=\left[\boldsymbol{\pi}_{b}, \boldsymbol{\pi}_{i},\right]$ be the stationary distribution, where $\boldsymbol{\pi}=\boldsymbol{\pi} \boldsymbol{D}$ and $\boldsymbol{\pi} \mathbf{1}=\mathbf{1}$. This is a realistic representation of the channel behavior under general conditions.

The behavior of the PU can be seen as an alternating sequence of busy and idle periods (white spaces). The duration of a white space follows a phase type distribution $(\boldsymbol{\omega}, W)$ [1], where $\boldsymbol{\omega}=\left(\boldsymbol{\pi}_{b} d_{b i} \mathbf{1}\right)^{-1} \boldsymbol{\pi}_{b} d_{b i}$ and $W=D_{i}$. Therefore, $\sigma_{k}$, the probability that the duration of uninterrupted white space is at least $k$ time slots, is given as

$$
\sigma_{k}=\frac{1}{\boldsymbol{\pi}_{b} d_{b i} \mathbf{1}} \boldsymbol{\pi}_{b} d_{b i} D_{i}^{k-1} \mathbf{1}, k \geq 1,
$$

and, $L_{w}$, the average duration of this white space is

$$
L_{w}=\sum_{k=1}^{\infty} \sigma_{k}=\frac{1}{\boldsymbol{\pi}_{b} d_{b i} \mathbf{1}} \boldsymbol{\pi}_{b} d_{b i}\left(I-D_{i}\right)^{-1} \mathbf{1} .
$$




\section{Model of the Cognitive Radio System}

In this section we consider the actions of the SUs and how they interact and/or co-exist with the PUs. As in the primary system, by SU we refer here to the whole set of SUs which might use the channel under consideration. Moreover we assume that all those SUs are coordinated. The SU can be in one of the following three situations: sleeping, sensing or transmitting.

Sleeping. The duration of the sleeping state is represented by the phase type distribution $(\boldsymbol{\delta}, L)$ of order $n_{\ell}$. After waking up the SU proceeds to the sensing cycle.

Sensing. In the sensing cycle the SU takes a series of measurements of the channel state in consecutive time slots. The maximum number of such measurements in a sensing cycle is described by the phase type distribution $(\boldsymbol{\beta}, S)$, of order $n_{s}$. If the outcome of any of the measurements is busy the sensing cycle is aborted and the SU goes back to sleep. Otherwise, if all measurements in the series find the channel idle, the SU proceeds to transmit.

Transmitting. The SU will transmit for a maximum number of slots represented by the phase type distribution $(\boldsymbol{\alpha}, T)$ of order $n_{t}$. Depending on the sensing characteristics and capabilities of the SU, it may, or may not, be able to detect the arrival of a PU during the transmitting state. In this study we assume that the SU interrupts immediately the transmission upon the arrival of a PU.

Let the source of SUs be saturated, i.e. there is always an SU looking for white space. We can easily consider the case of a non-saturated system. We also assume that the system gives preemptive priority to PUs over the SUs. In a separate paper later on we consider the case where we allow the system to keep track of the phase at which the SU was interrupted, and also relax the assumption about the source of SUs being saturated.

The time necessary to obtain a sensing measurement cannot be neglected compared to the transmission time of a data unit. The duration of a slot represents the length of time required to obtain a sensing measurement. The outcome of the sensing measurement performed during a slot is known at the end of the slot. In setting the result of the measurement we adopt a conservative approach: if any PU activity occurred during the slot the channel state will be deemed as busy, in other words the measurement undertaken during the time slot $[n, n+1)$ will yield idle as the result only if the PU was idle at both $t=n$ and $t=n+1$.

Despite the fact that now the sensing time cannot be neglected, it is assumed that sensing is carried out while the SU is transmitting. It could account for those situations where the SUs are equipped with required hardware to transmit and sense simultaneously or collaborative sensing is carried out and the gathered information is shared instantaneously among SUs. If we let the states of this system be classified as [(PU busy, SU sleeping), (PU busy, SU sensing), 
(PU idle, SU sleeping), (PU idle, SU sensing), and (PU idle, SU transmitting)], then we have the following discrete time Markov chain (DTMC),

$$
P=\left[\begin{array}{ccccc}
D_{b} \otimes L & D_{b} \otimes \boldsymbol{l} d_{b i} \otimes L & d_{b i} \otimes(\boldsymbol{l} \boldsymbol{\beta}) & 0 \\
D_{b} \otimes \boldsymbol{\delta} & 0 & d_{b i} \otimes \boldsymbol{\delta} & 0 & 0 \\
d_{i b} \otimes L & d_{i b} \otimes \boldsymbol{l} & D_{i} \otimes L & D_{i} \otimes(\boldsymbol{l} \boldsymbol{\beta}) & 0 \\
d_{i b} \otimes((S \mathbf{1}+\boldsymbol{s}) \boldsymbol{\delta}) & 0 & 0 & D_{i} \otimes S & D_{i} \otimes(\boldsymbol{s} \boldsymbol{\alpha}) \\
d_{i b} \otimes(\mathbf{1}) & 0 & 0 & 0 & D_{i} \otimes(T+\boldsymbol{t} \boldsymbol{\alpha})
\end{array}\right]
$$

where $\otimes$ denotes the Kronecker product [1, page 30] and $\boldsymbol{l}, \boldsymbol{s}$ and $\boldsymbol{t}$ are the absorption probability vectors associated with sleeping, sensing and transmitting phases, respectively.

This transition matrix captures the full behavior of this saturated system with preemptive discipline. Specifically, the throughput of the SUs can be extracted from this matrix after minor algebraic manipulations.

\subsection{Performance Measures}

We can now write the transition matrix as

$$
P=\left[\begin{array}{ccccc}
P_{11} & P_{12} & P_{13} & P_{14} & 0 \\
P_{21} & 0 & P_{23} & 0 & 0 \\
P_{31} & P_{32} & P_{33} & P_{34} & 0 \\
P_{41} & 0 & 0 & P_{44} & P_{45} \\
P_{51} & 0 & 0 & 0 & P_{55}
\end{array}\right]
$$

where the block elements $P_{i j}$ are as defined in the detailed matrix $P$.

Provided the Markov chain described by the matrix $P$ is irreducible then it has a stationary distribution $\boldsymbol{\pi}$ given as $\boldsymbol{\pi}=\boldsymbol{\pi} P, \boldsymbol{\pi} \mathbf{1}=1$, where $\boldsymbol{\pi}=$ $\left[\boldsymbol{\pi}_{1}, \boldsymbol{\pi}_{2}, \boldsymbol{\pi}_{3}, \boldsymbol{\pi}_{4}, \boldsymbol{\pi}_{5}\right]$.

Effective White Space Exceeds $\boldsymbol{K}$. Our interest here is in the state set 5 . We are interested in the probability that when the SU is finally able to transmit it has at least $K$ uninterrupted units of time slots for transmission without interfering with the PU. Let us further group the states as $A=\{1,2,3,4\}$ and $B=\{5\}$, and write the matrix $P$ as

$$
P=\left[\begin{array}{ll}
P_{A, A} & P_{A, B} \\
P_{B, A} & P_{B, B}
\end{array}\right],
$$

where $P_{B, B}=P_{55}$, and $\boldsymbol{\pi}_{B}=\boldsymbol{\pi}_{5}$ and the rest of the matrices and vectors are easily inferred from these two.

Then, $p_{K}$, the probability that the length of an effective white space is at least $K$ time slots can be written as

$$
p_{K}=\frac{1}{\boldsymbol{\pi}_{A} P_{A, B} \mathbf{1}} \boldsymbol{\pi}_{A} P_{A, B} P_{B, B}^{K-1} \mathbf{1} .
$$


Mean Effective White Space. From (2) it readily follows that, $L_{w}^{e}$, the mean effective white space is given as

$$
L_{w}^{e}=\sum_{k=1}^{\infty} p_{k}=\frac{1}{\boldsymbol{\pi}_{A} P_{A, B} \mathbf{1}} \boldsymbol{\pi}_{A} P_{A, B}\left(I-P_{B, B}\right)^{-1} \mathbf{1}
$$

Efficiency of the Sensing Strategy. We asses the efficiency of the sensing strategy from two different viewpoints:

- The effectiveness in using available white spaces for transmission; we represent this by $\eta_{t}$ and it is given as

$$
\eta_{t}=\frac{\boldsymbol{\pi}_{5} \mathbf{1}}{\boldsymbol{\pi}_{3} \mathbf{1}+\boldsymbol{\pi}_{4} \mathbf{1}+\boldsymbol{\pi}_{5} \mathbf{1}}
$$

which represents the fraction of slots available for SU transmission where it effectively transmits.

- The effectiveness in saving unnecessary measurements by remaining in the sleeping state while the PU is busy; we represent this by $\eta_{s}$ and it is given as

$$
\eta_{s}=\frac{\boldsymbol{\pi}_{1} \mathbf{1}}{\boldsymbol{\pi}_{1} \mathbf{1}+\boldsymbol{\pi}_{2} \mathbf{1}}
$$

which represents the fraction of slots the $\mathrm{SU}$ is in sleeping state while the PU is busy. Saving as much measurements as possible, i.e. a high value of $\eta_{s}$, is a desirable characteristic of the sensing strategies as it means saving energy or being able to use the radio to sense other channels.

Obviously, we have that $0 \leq \eta_{t}, \eta_{s} \leq 1$. Besides, there is trade-off between these two efficiency measures. Short sleeping periods leads to high values of $\eta_{t}$ and low value of $\eta_{s}$, and vice versa. We summarize both measures into an overall efficiency factor, $\eta$, as

$$
\eta=\alpha \eta_{t}+(1-\alpha) \eta_{s}
$$

where $0 \leq \alpha \leq 1$. Note that $\eta \leq \alpha \max \left(\eta_{t}, \eta_{s}\right)+(1-\alpha) \max \left(\eta_{t}, \eta_{s}\right)=\max \left(\eta_{t}, \eta_{s}\right)$, and analogously $\eta \geq \min \left(\eta_{t}, \eta_{s}\right)$. Hence, $0<\min \left(\eta_{t}, \eta_{s}\right) \leq \eta \leq \max \left(\eta_{t}, \eta_{s}\right)<1$.

\section{Experimental Results and Discussion}

In this section we use the model to assess different types of sensing strategies. By sensing strategies we imply that, given a fixed mean sleeping interval, we want to design rules for sleeping that can achieve this selected mean interval. We then assess which one of them performs the best. Specifically, we consider the following: deterministic, uniform, geometric and negative binomial (or Pascal). All these distributions can be represented by discrete phase type distributions [1]. 


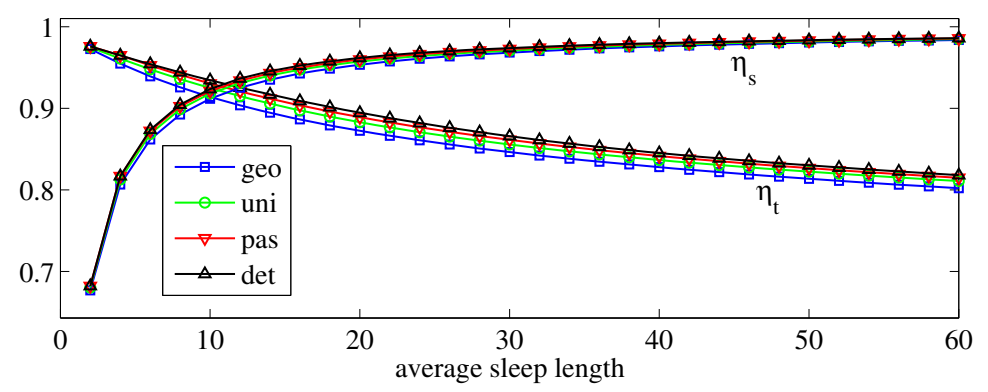

Fig. 1. Efficiency of different sensing strategies. Lightly loaded channel, $\rho_{P U}=0.2$.

For the channel occupancy we use the model proposed in [7, which is shown to exhibit a self-similar behavior over a finite but wide enough range of time-scales. Thus the block-matrices in (1) are given as

$$
D=\left[\begin{array}{c|cccc}
1-\sum_{k=0}^{n-1} \frac{1}{a^{k}} & \frac{1}{a} & \frac{1}{a^{2}} & \cdots & \frac{1}{a^{n-1}} \\
\hline \frac{b}{a} & 1-\frac{b}{a} & & \\
\left(\frac{b}{a}\right)^{2} & & 1-\left(\frac{b}{a}\right)^{2} & & \\
\vdots & & & \ddots & \\
\left(\frac{b}{a}\right)^{n-1} & & & & 1-\left(\frac{b}{a}\right)^{n-1}
\end{array}\right],
$$

where $n, a$ and $b$ are the parameters of the model. The value of $n$ determines the range of time-scales where the process can be considered as self-similar. Once the value of $n$ is set the values of $a$ and $b$ are obtained so as to fit the utilization factor of the channel $\rho_{P U}=(1-1 / b) /\left(1-1 / b^{n}\right)$, and the average number of consecutive slots that the channel is busy (avg. burst length) $E[B]=\left(\sum_{k=0}^{n-1} a^{-k}\right)^{-1}$. In all the numerical results shown here we set $n=8$ and $E[B]=20$.

The SU is in the transmitting phase for a maximum number of slots that follows a geometric distribution with mean value equal to 10 . The maximum duration of a sensing cycle is deterministically set to 2 slots. In what follows we study the impact on the efficiency of varying the distribution type and the mean value for the duration of the sleeping phase.

The impacts of the sensing strategy on both $\eta_{t}$ and $\eta_{s}$ are shown in Fig. 1. As expected by spending longer intervals in the sleeping state $\eta_{t}$ decreases and $\eta_{s}$ increases. Note however that $\eta_{s}$ increases abruptly at first, reaches rather high values and then levels off. Comparatively, the counter decline in $\eta_{t}$ occurs more gradually. The observed trends suggest that weighted efficiency $(\eta)$ will attain a peak for short durations of the sleep period, as it is confirmed in Fig. 2. We also observe that as the channel gets more loaded the value of the optimal efficiency decreases and, more importantly, the proper adjustment of the sleep duration becomes a more sensitive issue.

In the comparison of different distributions for the sleep duration (keeping constant the mean value) it was observed that lower variability results in better 


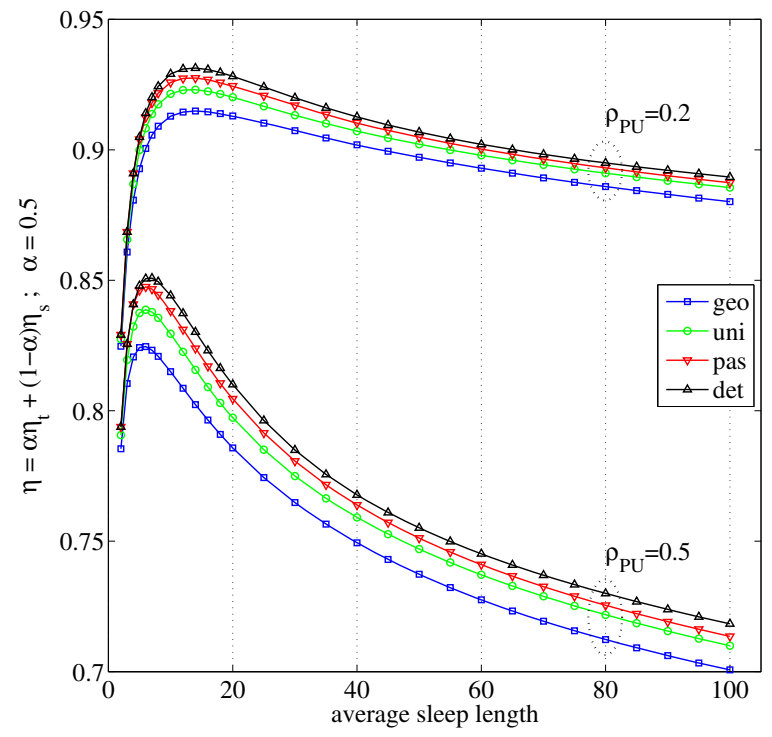

Fig. 2. Overall efficiency of different sensing strategies. Lightly loaded channel, $\rho_{P U}=$ 0.2 ; moderately loaded channel, $\rho_{P U}=0.5$.

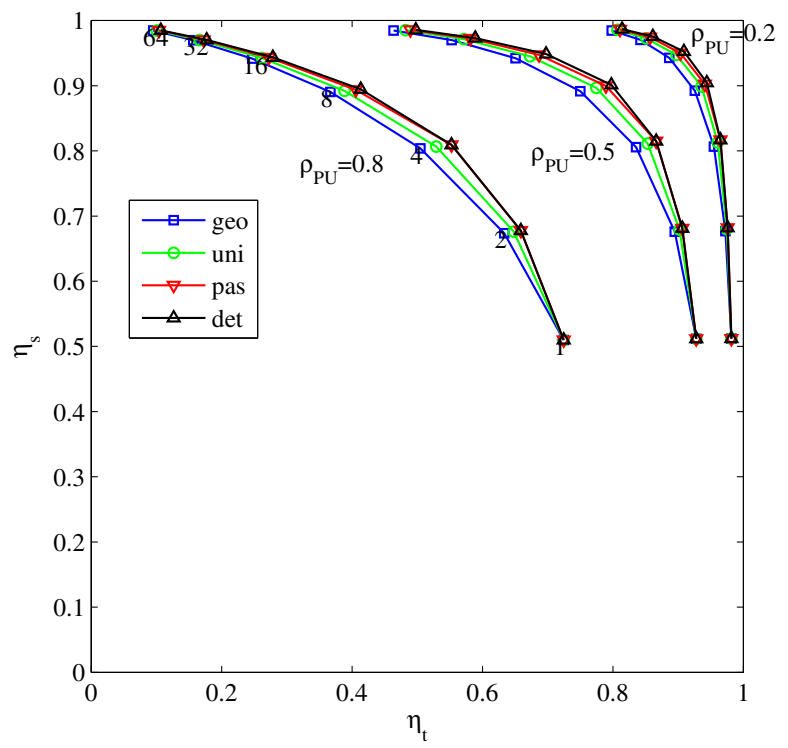

Fig. 3. Trade-off between $\eta_{t}$ and $\eta_{s}$. The numbers next to the points denote the average sleep length for the nearby set of points. 


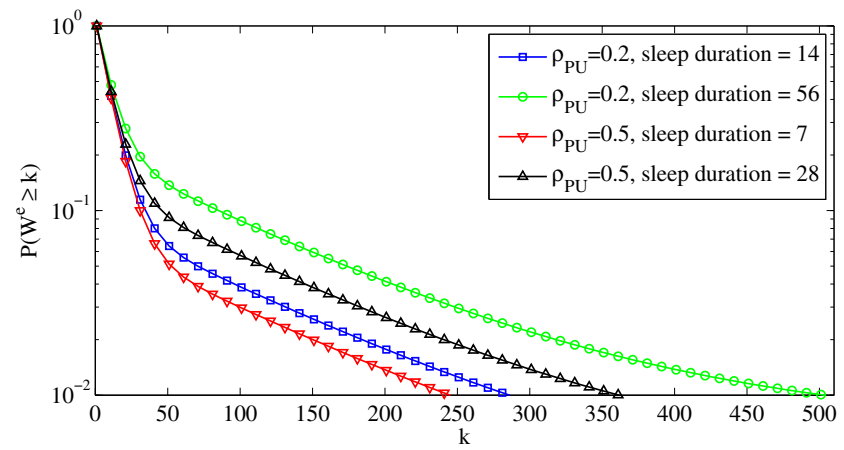

Fig. 4. Complementary CDF of the duration of an effective white space. Deterministic sleep duration.

efficiency — in all our experiments the deterministic distribution yield the highest overall efficiency - although differences are very small. The efficiency trade-off is also explored in Fig. 3] where $\eta_{s}$ and $\eta_{t}$ are displayed one versus the other. There are three bundles of curves that correspond to different channel utilizations (high, $\rho_{P U}=0.8$; moderate, $\rho_{P U}=0.5$; low, $\rho_{P U}=0.2$ ). The four curves in each bundle correspond to the four distributions of the sleep time, and along each curve the mean sleep time is varied. For a given value of the mean sleep time the value of $\eta_{s}$ is approximately the same independently of the channel load. However, as the load increases the curves stretch on the horizontal dimension, which means that a higher cost in terms of $\eta_{t}$ has to be paid to achieve a high value of $\eta_{s}$.

The impact of the sensing strategy on the distribution of the duration of an effective white space is shown in Fig. 4. Other experiments - not shown here due to the lack of space- shown that differences across distributions were not noticeable for this type of representation. Thus on Fig. 4 we only represented the results for the deterministic distribution of sleep length. For each channel load two different lengths are represented: the one at which the weighted efficiency peaks in Fig. 2 and another one which is four times larger.

\section{Conclusion}

In this paper we have established, through an analytical model, that constant interval sensing is more effective than all the other strategies tested. This is as expected. The model used is designed for general application for studying the behavior of cognitive radio networks. In the next stage of our work we plan to use this model to study the waiting time for an SU to obtain a particular length of white space and several other performance measures. 


\section{References}

1. Alfa, A.S.: Queueing Theory for Telecommunications: Discrete Time Modelling of a Single Node System. Springer, Heidelberg (2010)

2. FCC: Spectrum Policy Task Force Report. Tech. Rep. ET Docket 02-135, Federal Communications Commission (November 2002)

3. Geirhofer, S., Tong, L., Sadler, B.: Dynamic spectrum access in the time domain: Modeling and exploiting white space. IEEE Communications Magazine 45(5), 66-72 (2007)

4. Ghasemi, A., Sousa, E.: Spectrum sensing in cognitive radio networks: requirements, challenges and design trade-offs. IEEE Communications Magazine 46(4), 32-39 (2008)

5. Hoang, A., Liang, Y., Wong, D., Zeng, Y., Zhang, R.: Opportunistic spectrum access for energy-constrained cognitive radios. IEEE Transactions on Wireless Communications 8(3), 1206-1211 (2009)

6. Lee, W.Y., Akyildiz, I.: Optimal spectrum sensing framework for cognitive radio networks. IEEE Transactions on Wireless Communications 7(10), 3845-3857 (2008)

7. Robert, S., Le Boudec, J.: New models for self-similar traffic. Performance Evaluation 30(1-2), 57-68 (1997)

8. Wellens, M., Riihijärvi, J., Mähönen, P.: Empirical time and frequency domain models of spectrum use. Physical Communication 2(1-2), 10-32 (2009)

9. Yucek, T., Arslan, H.: A survey of spectrum sensing algorithms for cognitive radio applications. IEEE Communications Surveys \& Tutorials 11(1), 116-130 (2009) 